\title{
Association between methylation in nasal epithelial TSLP gene and chronic rhinosinusitis with nasal polyps
}

Jingyun Li ${ }^{1,2}$, Jian Jiao ${ }^{1,2}$, Yunbo Gao ${ }^{1,2}$, Yuan Zhang ${ }^{1,2,3^{*}}$ and Luo Zhang ${ }^{1,2,3^{*}}$ (D)

\begin{abstract}
Background: This study was performed to determine whether there was any association between abnormal DNA methylation of a thymic stromal lymphopoietin (TSLP) locus and pathogenesis of chronic rhinosinusitis (CRS).

Methods: A total of 48 CRS patients with nasal polyps (CRSwNP), 28 CRS patients without nasal polyps (CRSsNP) and 21 control subjects were enrolled into the study; and evaluated for serum total IgE level, olfactory score and nasal resistance. Samples were obtained from nasal polyps of CRSwNP patients, ethmoid mucosae of CRSsNP patients and inferior turbinate (IT) mucosa of control subjects during surgery, and used to isolate purified primary human nasal epithelial cells (HNECs). Genomic DNA was extracted from purified primary HNECs of each subject and DNA methylation ratios for a selected region of the TSLP gene were screened the using MasSARRAY EpiTYPER.
\end{abstract}

Results: A total of $17 \mathrm{CpG}$ units were analyzed; of which two CpG units (CpG3 and 22:23:24) had increased methylation ratios in the CRSWNP patients compared to the CRSsNP and control subjects after correction for false discovery rate $(F D R)(Q<0.1)$. The methylation ratios at both $\mathrm{CpG} 3$ and $\mathrm{CpG} 22: 23: 24$ units were positively correlated with olfactory score $(r=0.41, P=0.0001 ; r=0.25, P=0.021)$ and unilateral nasal resistance at $75 \mathrm{~Pa}(r=0.24, P=0.04$; $r=0.24, P=0.036)$ and $150 \mathrm{~Pa}(r=0.34, P=0.004 ; r=0.25, P=0.031)$. Total nasal resistance at $75 \mathrm{~Pa} / 150 \mathrm{~Pa}$ or serum total IgE levels were not correlated with the methylation ratios at either CpG unit.

Conclusions: Increased DNA methylation at the TSLP locus is likely to be associated with CRSwNP pathogenesis; however these findings need to be confirmed in larger multicentre group studies.

Keywords: Chronic rhinosinusitis, DNA methylation, Nasal resistance, Olfactory score, Thymic stromal lymphopoietin (TSLP)

\section{Background}

Chronic rhinosinusitis (CRS) is one of the most common inflammatory disorders of the sinus and paranasal sinus mucosa, which affects approximately $5-15 \%$ of the general population worldwide [1-3]. CRS can generally be classified into two major phenotypes; including CRS with nasal polyps (CRSwNP) and CRS without nasal polyps (CRSsNP). Although CRS is a highly complex

\footnotetext{
*Correspondence: summer_zhang1211@126.com; dr.luozhang@139.com

${ }^{3}$ Department of Allergy, Beijing TongRen Hospital, Capital Medical

University, Beijing 100176, China

Full list of author information is available at the end of the article
}

disease, both genetic and environmental factors are believed to contribute to the pathogenesis of this disease.

Epigenetic modification is essential for regulating tissue and stage-specific gene expression during growth and development, and can serve as a mediator of geneenvironment interactions in the pathogenesis and progression of disease [4]. DNA methylation is one of the most investigated and important forms of epigenetic modification, and involves the covalent addition of a methyl group to the fifth carbon of a cytosine $(C)$ residue, mostly in the context of CG dinucleotide $(\mathrm{CpG})$. Clusters of these CpG sites (CpG island) are located in the promoter regions of $50 \%$ genes [5]. Although hypermethylation of $\mathrm{CpG}$ sites at gene promoter regions 
is commonly thought to be related to transcriptional silencing, some recent studies have demonstrated that hypermethylation of CpG residues was significantly associated with activation of some genes [6, 7]. It has been shown that alteration of DNA methylation may contribute to some mechanisms relevant to CRS pathophysiology, including transcriptional regulation of some $\mathrm{T}_{\mathrm{H}}$ genes and cytokine production [8].

Thymic stromal lymphopoietin (TSLP) is an IL-7 like and epithelial cell derived cytokine that is commonly recognized as a driver of $\mathrm{T}_{\mathrm{H}} 2$ responses. TSLP stimulates dendritic cells $(\mathrm{DCs})$ to induce naïve $\mathrm{CD} 4^{+} \mathrm{T}$ cell differentiation into $\mathrm{T}_{\mathrm{H}} 2$ cells and cytokine production [9], indicating that TSLP plays an important role in allergic and immune disorders. Indeed, some studies have shown that TSLP may be involved in the aetiology of CRS [10-12]. While one study has demonstrated significant overexpression of TSLP mRNA in NP tissues from CRSwNP patients compared to nasal mucosa from CRSsNP and control subjects [10], another study has shown that the numbers of immunohistochemically stained $\mathrm{TSLP}^{+}$nasal epithelial cells were significantly increased in NP tissues from atopic subjects compared to non-atopic subjects and also strongly correlated with the number of tissue eosinophils and IgE levels in NP tissue [11]. Similarly, studies investigating genetic polymorphisms in the TSLP gene have reported specific single nucleotide polymorphisms (SNPs) to be linked with asthma [13], atopic dermatitis (AD) [14] and allergic rhinitis (AR) [15]. We have previously demonstrated that SNPs in the TSLP gene exhibit a gender and/or nasal polyps-dependent risk for CRS [16].

To our knowledge, the epigenetic change/s in TSLP gene involved in the pathogenesis of CRS is/are presently not well understood. Thus, the aim of the present study was to investigate any relationship between abnormal DNA methylation status of a CpG island in the TSLP gene and pathogenesis of CRS with or without nasal polyps.

\section{Methods \\ Subjects}

The study protocol was approved by the Ethics Committee of Beijing Tongren Hospital and all subjects provided written informed consent prior to recruitment. A total of 48 patients with CRSwNP and 28 patients with CRSsNP, fulfilling the criteria of CRS according to the European Position Paper on Rhinosinusitis and Nasal Polyps (EPOS) 2012 guidelines [17] were recruited from the Rhinology Department of Beijing Tongren Hospital, from November 2014 to November 2015. The diagnosis of CRS was confirmed by trained rhinologists, based on patients' histories, clinical examinations, nasal endoscopies and computed tomographic scans of the sinuses. A total of 21 subjects, who demonstrated no sinonasal disease by rhinoscopy and presented for septoplasty for anatomic variations, were recruited to the study as control subjects.

None of the subjects had used oral or nasal corticosteroids for 4 weeks or antibiotics for 2 weeks before their surgeries. Atopic status was confirmed by detecting IgE antibodies against various common inhalant allergens using the Phadiatop test (Phadia, Uppsala, Sweden). Serum total IgE level was detected using the ImmunoCAP 100 system (Pharmacia, Uppsala, Sweden). Diagnosis of asthma was made by pulmonologists, based on lung function and allergen challenge tests. Nasal polyps score for each subject was evaluated by a different observer using the Davos scoring system. The olfactory function test was performed using the Toyota and Takagi (T\&T) olfactometer (Daiichi Pharmaceutical Co. Ltd., Tokyo, Japan), according to the manufacturer's instructions. The score was assessed as the average of the sum of odor threshold for 5 odorants. For the T\&T test, a score of 1 was defined as euosmia, $2=$ mild hyposmia, $3=$ moderate hyposmia, $4=$ severe hyposmia and $5=$ anosmia. Nasal resistance was measured at $75 \mathrm{~Pa}$ and $150 \mathrm{~Pa}$, respectively, by HRR2 four-phase rhinomanometry (Rhino Lab GmbH, Rendsburg, Germany), as described previously [18]. The left (RL) and right (RR) lateral nasal resistance values were calculated according to Ohm's law; and the total nasal resistance was calculated using the formula $(\mathrm{RL} \times \mathrm{RR}) /(\mathrm{RL}+\mathrm{RR})$, and the unilateral nasal resistance calculated usung the formula $(R L+R R) / 2$.

Tissue samples were obtained from nasal polyps of patients with CRSwNP, ethmoid mucosa of patients with CRSsNP, and inferior turbinate (IT) mucosa of control subjects during surgery. Primary human nasal epithelial cells (HNECs) were derived from these above tissues as previously described [19]. Briefly, the tissue sample was dissociated enzymatically by incubation overnight in medium containing $0.1 \%$ protease and the isolated purified epithelial cells were collected by centrifugation at $120 \mathrm{~g}$ for $10 \mathrm{~min}$ for analysis of DNA methylation.

\section{DNA methylation analysis by SequenomMassARRAY EpiTYPER}

Genomic DNA was extracted from purified primary HNECs of each subject using human tissue DNA kit (Omega Bio-tek, Georgia, CA, USA). DNA quality was assessed by electrophoretic migration on $1 \%$ agarose gel, and the DNA concentration was measured by UV spectroscopy at $260 \mathrm{~nm}$. A total of $1 \mu \mathrm{g}$ genomic DNA was bisulfite-treated with EZ DNA methylation kit (Zymo Research, Orange, CA, USA), and the 
SequenomMassARRAY EpiTYPER platform (Sequenom, San Diego, CA, USA) was used to detect the DNA methylation ratios for a selected region in each sample, according to the manufacturer's instructions. The EpiDesigner software (Sequenom, San Diego, CA, USA) was designed to systematically screen the methylation status of $29 \mathrm{CpG}$ sites in the TSLP gene. Due to the cleavage pattern, some $\mathrm{CpG}$ sites were analysed in units. The sequence and location of the $\mathrm{CpG}$ islands are shown in Fig. 1 and Additional file 1: Figure S1. The positions and sequences of primers used for the EpiTYPER assay to analyze DNA methylation of TSLP locus are shown in Additional file 1: Table S1.

\section{Statistical analysis}

The demographic and clinical characteristics of the study population were expressed as proportions or mean and range, and differences between the three study groups were evaluated by $x^{2}$ test for categorical variables, oneway ANOVA and Kruskal-Wallis test for continuous variables.

Differences in methylation ratios among CRSsNP, CRSwNP and Control subjects were analysed using nonparametric Kruskal-Wallis test followed by a
Mann-Whitney $U$ test. The false discovery rate (FDR) was used to correct for multiple testing for the $\mathrm{CpG}$ sites; with a $\mathrm{Q}<0.1$ considered to be significant. Linear regression modelling was used to evaluate the relationship between methylation ratios of associated CpG units and disease status after adjustment for age, gender, smoking, drinking and comorbid asthma and allergic status. Spearman correlation test was used for assessing the presence of any correlation between the methylation status of a CpG unit and clinical factors. SPSS 22.0 software for Windows was used for data analysis. A value of $P<0.05$ was considered statistically significant.

\section{Results}

Demographic and clinic characteristics of the subjects

The demographic and clinic characteristics of the subjects in the three study groups are summarized in Table 1. Overall, the three groups were matched for ethnicity and geographical location and had similar ratios of gender, smoking and drinking status, the prevalence of comorbid allergy and asthma, and the mean serum total IgE levels. The two CRS subgroups had significantly older subjects, with higher olfactory scores and total and unilateral nasal

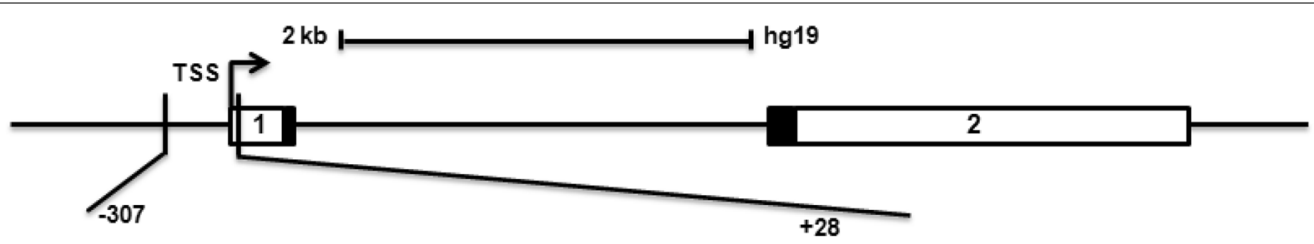

CGtgcagaactcCGagaggtgcctgggctcCGggaCGcCGcCGcCGggggaaa \begin{tabular}{llll}
1 & 2 & 3 & $4: 5: 6: 7$ \\
\hline
\end{tabular}

ggggacatctgggctgtcagagCGgggctgCGcctagcttgggacaacacttctgttcc 8:9

aatttagggagaggaagtctctatc $C$ Ggaggaaaggcaaattgggaactggga $C$ Gagg 1011

gaaCGttgttaggggcaccacctgctggggtcCGgCGcctcCGCGctCGggetCG \begin{tabular}{|llll}
\hline 12 & $13: 14$ & $15: 16$ & 17 \\
\hline
\end{tabular} gaattttggcagcctcCGcccetggagacttgggaggagCGagCGtgggtgacagtc 19 20:21 ttttCGCGaCGagtgccetcCGccacctCGccaCGccetgetccccCGCG

\begin{tabular}{|lll}
\hline $22: 23: 24$ & 25 & $26: 27$ \\
\hline
\end{tabular}

Fig. 1 Genomic localization and overview of the CpG sites investigated. In the present study, a large region in a CpG island located in the sfTSLP proximal promoter was covered from -307 to +28 . CpG11 site could not be measured because of technical limitations of the assay. TSS transcription start site 
Table 1 Demographic and clinical characteristics of study participants

\begin{tabular}{|c|c|c|c|c|}
\hline Characteristic & Control subjects & Patients with CRSsNP & Patients with CRSwNP & $P$ \\
\hline No. & 21 & 28 & 48 & \\
\hline Gender (female/male) & $12 / 9$ & $21 / 7$ & $35 / 13$ & 0.34 \\
\hline Age (years), median (range) & $44(18-65)$ & $46.5(19-68)$ & $56(18-67)$ & 0.02 \\
\hline Allergy, no (\%) & $1(4.8)$ & $7(25)$ & $11(22.9)$ & 0.15 \\
\hline Asthma, no (\%) & $0(0)$ & $0(0)$ & $5(10.4)$ & 0.07 \\
\hline Smoking, no (\%) & $5(23.8)$ & $7(25)$ & $18(37.5)$ & 0.38 \\
\hline Drinking, no (\%) & $6(28.6)$ & $7(25)$ & $17(35.4)$ & 0.61 \\
\hline Serum total lgE (kU/l), median (range) & $43(2-410)$ & $83.95(6.04-577)$ & $48(2.59-1017)$ & 0.38 \\
\hline Polyp score (Davos), median (range) & 0 & 0 & $3(1-6)$ & $<0.001$ \\
\hline Prior nasal surgeries, no (\%) & $0(0)$ & $0(0)$ & $12(25.0)$ & $<0.001$ \\
\hline Olfactory score, median (range) & 1 & $1(1-5)$ & $4(1-5)$ & 0.003 \\
\hline Total nasal resistance $\left(\mathrm{Pa} / \mathrm{cm}^{3} / \mathrm{s}\right), 75 \mathrm{~Pa}$, median (range) & $0.116(0-0.301)$ & $0.145(0.095-0.357)$ & $0.073(0-8.163)$ & 0.041 \\
\hline Unilateral nasal resistance $\left(\mathrm{Pa} / \mathrm{cm}^{3} / \mathrm{s}\right), 75 \mathrm{~Pa}$, mean (range) & $0.276(0.150-0.685)$ & $0.373(0.200-0.737)$ & $0.463(0.141-16.811)$ & 0.001 \\
\hline Total nasal resistance $\left(\mathrm{Pa} / \mathrm{cm}^{3} / \mathrm{s}\right), 150 \mathrm{~Pa}$, median (range) & $0.139(0-0.354)$ & $0.190(0-0.441)$ & $0.247(0-11.768)$ & 0.045 \\
\hline Unilateral nasal resistance $\left(\mathrm{Pa} / \mathrm{cm}^{3} / \mathrm{s}\right), 150 \mathrm{~Pa}$, mean (range) & $0.303(0.199-0.797)$ & $0.507(0.244-2.371)$ & $0.614(0.121-23.703)$ & 0.001 \\
\hline
\end{tabular}

Italic values indicate significance of $P$ value $(P<0.05)$

resistance, than the control group. A total of 12 patients with CRSwNP (25\%) had prior nasal surgeries, compared to none of the control subjects or patients with CRSsNP.

\section{DNA methylation in TSLP gene in primary HNECs}

TSLP gene is present as two transcript variants coding two distinct isoforms, the long- and short-form TSLP (IfTSLP and sfTSLP, respectively). Based on the CpG island track profiles of UCSC Genome Browser (genome. ucsc.edu), TSLP gene contains one CpG island harboring a total of $29 \mathrm{CpG}$ residues, which locates in the sfTSLP proximal promoter while in the second intron of lfTSLP gene (Fig. 1 and Additional file 1: Figure S1).

A total of $17 \mathrm{CpG}$ units were analyzed. As shown in Fig. 2, in the CRSwNP patients, two CpG units (CpG3 and 22:23:24) had increased methylation ratios compared to the CRSsNP and control subjects after correction for FDR $(\mathrm{Q}<0.1)$.

To exclude the effect of confounder factors on the association between methylation ratios of CpG3 and CpG22:23:24 units and CRSwNP, we performed linear regression models and found that these significant relationships remained robust even after adjusting

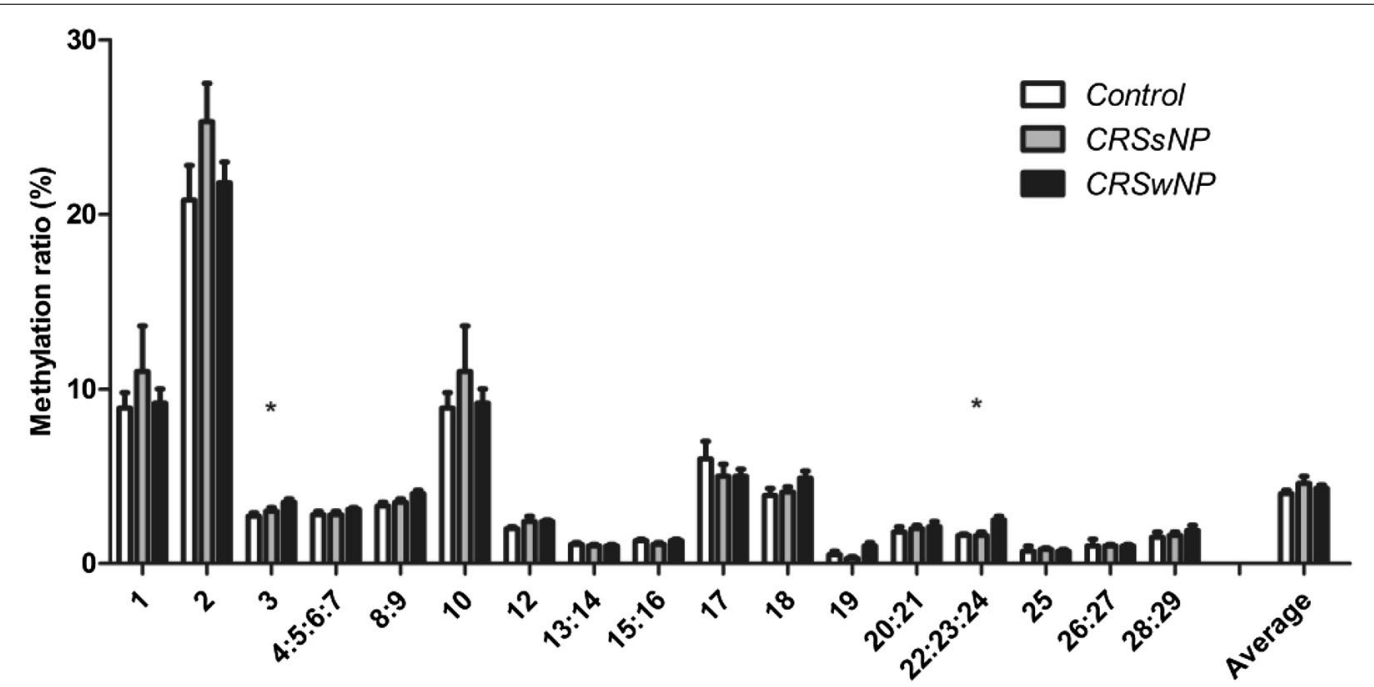

Fig. 2 Comparison of methylation ratios at individual CpG residues among CRSsNP and CRSwNP patients and control subjects. Results are expressed as mean \pm SEM. FDR was used for multiple testing; with ${ }^{*}=Q<0.1$ 
for potential confounders including age, gender, smoking, drinking and comorbid asthma and allergic status (Table 2). However, greater age was significantly associated with increased methylation ratios of $\mathrm{CpG} 3$, but not with increased methylation ratios of CpG22:23:24 in the TSLP promoter (Table 2).

\section{Impact of DNA methylation in TSLP gene on clinical factors} Both methylation ratios at CpG3 and CpG22:23:24 units were positively correlated with olfactory score $(\mathrm{r}=0.41$, $P=0.0001 ; \mathrm{r}=0.25, P=0.021)$ as well as unilateral nasal resistance at $75 \mathrm{~Pa}(\mathrm{r}=0.24, P=0.04 ; \mathrm{r}=0.24, P=0.036)$ and $150 \mathrm{~Pa}(\mathrm{r}=0.34, P=0.004 ; \mathrm{r}=0.25, P=0.031)$, but not correlated with total nasal resistance at $75 \mathrm{~Pa}$ or $150 \mathrm{~Pa}$, nor serum total IgE levels (Table 3).

\section{Discussion}

Most of the studies on the role of TSLP in the pathogenesis of CRS to date have focused on nonepigenetic and genetic mechanisms; with relatively little known on the epigenetic mechanisms involved. The present study employed MassARRAY EpiTYPER technique to widely screen the methylation status of a CpG island in the TSLP gene and then evaluated any associations between the methylation ratios of these $\mathrm{CpG}$ units and CRS. We found that the methylation ratios of two CpG units, CpG3 and CpG22:23:24 were strongly associated with susceptibility to CRSwNP. Furthermore, analysis of clinical symptoms showed positive

\begin{tabular}{|c|c|c|c|}
\hline Factors & $\begin{array}{l}\text { Standardized } \\
\text { beta }\end{array}$ & $95 \% \mathrm{Cl}$ & $P$ \\
\hline \multicolumn{4}{|l|}{ CpG3 } \\
\hline Disease status & 0.26 & $0.001-0.006$ & 0.019 \\
\hline Gender & -0.11 & $-0.008-0.003$ & 0.38 \\
\hline Age & 0.25 & $2.6 \mathrm{E}-5-3.8 \mathrm{E}-4$ & 0.025 \\
\hline Smoking & -0.13 & $-0.009-0.003$ & 0.32 \\
\hline Drinking & -0.01 & $-0.006-0.006$ & 0.92 \\
\hline Comorbid asthma & 0.08 & $-0.007-0.014$ & 0.47 \\
\hline Allergic disease & -0.03 & $-0.006-0.005$ & 0.77 \\
\hline \multicolumn{4}{|l|}{ CpG22:23:24 } \\
\hline Disease status & 0.31 & $0.002-0.009$ & 0.006 \\
\hline Gender & 0.04 & $-0.006-0.009$ & 0.73 \\
\hline Age & 0.08 & $-1.5 \mathrm{E}-4-3.3 \mathrm{E}-4$ & 0.48 \\
\hline Smoking & -0.16 & $-0.01-0.003$ & 0.23 \\
\hline Drinking & 0.15 & $-0.004-0.013$ & 0.27 \\
\hline Comorbid asthma & -0.17 & $-0.02-0.002$ & 0.10 \\
\hline Allergic disease & -0.04 & $-0.009-0.006$ & 0.72 \\
\hline
\end{tabular}

Italic values indicate significance of $P$ value $(P<0.05)$ correlations between the methylation ratios of $\mathrm{CpG} 3$ and CpG22:23:24 and olfactory score and unilateral nasal resistance, respectively. Indeed, it is possible that the association the methylation ratios of CpG3 and CpG22:23:24 and olfactory score may partly be agerelated because greater age was significantly associated with increased methylation ratio of particularly $\mathrm{CpG} 3$ and because olfactory function declines with age.

TSLP has pleiotropic biological and pathological functions;with overexpression of TSLP detected in several allergic diseases, including asthma, AD, AR and food allergy [20]. In the thymus, TSLP is expressed by Hassal corpuscles and regulates the capacity of dendritic cells(DCs) and plasmacytoid DCs to promote the differentiation of natural regulatory $\mathrm{T}$ cells [21, 22]. Indeed, one study demonstrated that TSLP-activated DCs induced a robust homeostatic polyclonal expansion of T cells [23]. Moreover, TSLP has been shown to induce a strong $\mathrm{T}_{\mathrm{H}}$ 2-mediated inflammation by increased production of numerous $\mathrm{T}_{\mathrm{H}} 2$ cytokines; such as IL-4, IL-5 and IL-13 as well as TNF- $\alpha$ [9], through upregulation of OX40 ligand expression on TSLP-activated DCs [24]. Similarly, TSLP levels have been shown to be significantly increased in CRSwNP nasal mucosa compared to CRSsNP and control nasal mucosa; and furthermore positively associated with markers of $\mathrm{T}_{\mathrm{H}} 2$ responses and eosinophilia [10-12]. These findings suggest that elevated levels of TSLP may be involved in the pathogenesis of CRSwNP through the regulation of $\mathrm{T}_{\mathrm{H}} 2$ inflammatory cytokine accumulation. A genetic study from our laboratory has previously shown that the TSLP gene may be important in conferring susceptibility to CRS, especially CRSwNP [16]. While some polymorphisms in or near the TSLP gene have been reported to be associated with multiple allergic diseases [13-15], the specific mechanism underlying the involvement of these polymorphisms has yet to be revealed. Thus, the present study was undertaken with the view that investigation of

\begin{tabular}{|c|c|c|c|c|}
\hline \multirow[t]{2}{*}{ Characteristic } & \multicolumn{2}{|l|}{ CpG3 } & \multicolumn{2}{|c|}{ CpG22:23:24 } \\
\hline & $r$ & $P$ & $r$ & $P$ \\
\hline Serum total lgE & 0.16 & 0.23 & -0.042 & 0.75 \\
\hline Olfactory score & 0.41 & 0.0001 & 0.25 & 0.021 \\
\hline Total nasal resistance, $75 \mathrm{~Pa}$ & 0.095 & 0.416 & 0.13 & 0.26 \\
\hline Unilateral nasal resistance, $75 \mathrm{~Pa}$ & 0.24 & 0.04 & 0.24 & 0.036 \\
\hline Total nasal resistance, $150 \mathrm{~Pa}$ & 0.17 & 0.16 & 0.13 & 0.28 \\
\hline Unilateral nasal resistance, $150 \mathrm{~Pa}$ & 0.34 & 0.004 & 0.25 & 0.031 \\
\hline
\end{tabular}


methylation status of the TSLP gene might provide better understanding of a role of TSLP gene in CRS.

TSLP has two distinct isoforms (long and short) which are encoded by two different transcript variants [25]. The canonical variant encodes the longer isoform (known as lfTSLP), which is the type thought to promote allergic inflammation in diseases. Similarly, the transcript variant 2 encodes the shorter isoform (known as sfTSLP), which is reported to play an antimicrobial role [26]. Data on gene structure of human TSLP transcripts in the UCSC Genome Browser shows that there are two putative promoter regions to activate the two TSLP transcript variants. The ENCODE track profiles show that the short isoform promoter, which covers the CpG island investigated in the present study, has a high capacity to bind multiple different transcription factors. It is possible that methylation at specific $\mathrm{CpG}$ sites may modify these sites and therefore influence the affinity for transcription factors near the cis-elements. In the present study we found that overall methylation ratios at the sfTSLP promoter were not significantly different between HNECs of CRSsNP and CRSwNP patients. In contrast, a study by Luo and colleagues [27] has reported that average methylation at the remote promoter of IfTSLP was significantly decreased in skin lesions from patients with AD compared with healthy controls. It is possible that the discordance between our study and that of Luo and colleagues may be due to differences in the $\mathrm{CpG}$ sites assessed for in the promoter regions of sfTSLP and IfTSLP, and differences in the cell types investigated. However, comparison of the difference in methylation ratios at individual $\mathrm{CpG}$ sites or units among CRSwNP, CRSsNP or controls in the present study has demonstrated that there was significantly increased of DNA methylation at the CpG3 and CpG22:23:24 sites in CRSwNP patients. While the precise role of hypermethylation of the CpG3 and CpG22:23:24 sites is presently not clear, it is possible that CpG3 and CpG22:23:24 hypermethylation may act as a positive regulatory mechanism of IfTSLP; particularly as CHIP-seq experiments from ENCODE track have exhibited binding of some cis-acting transcriptional repressors such as CTBP2 and CTCF in this region. Thus, hypermethylation at CpG3 and CpG22:23:24 may be a mechanism to disrupt binding of these transcriptional repressors to these sites. On the other hand, it is possible that CpG3 and CpG22:23:24 hypermethylation may involve the regulation of sfTSLP; particularly as sfTSLP has an opposite immune function compared with IfTSLP. This may be important as upregulation of sfTSLP is one of the most important mechanisms of vitamin D-mediated protection against the airway inflammation $[25,28]$. Recently, Lan and colleagues [29] reported that
sfTSLP was decreased in CRSwNP tissue after S. aureus infection, suggesting sfTSLP may participate in the nasal mucosal defence against bacterial infection. In the present study, the effect of several potential confounders; including age, gender, smoking, drinking and comorbid asthma and allergic status were also taken into account, and after adjusting for these factors, hypermethylation at CpG3 and CpG22:23:24 was still found to be associated with CRSwNP. Although our results suggest that the methylation status of TSLP may be a key independent factor and implicate hypermethylation of TSLP DNA in increased susceptibility to CRSwNP, presently these findings do not provide any clear indications with regard to the specific mechanism/s involved in this aspect.

Sensorineural olfactory loss may lead to the hyposmia in CRS patients. Local increase of inflammatory cytokines such as TNF- $\alpha$ and INF- $\gamma$ can suppress the olfactory neuron function, turnover and survival [30-32]. Similarly, TSLP also have neurotoxic potential, and the epithelial cells in the airway can directly communicate to cutaneous sensory neurons via TSLP to promote itch [33]. Thus, it is conceivable that TSLP may be modulated in olfactory dysfunction in CRS patients. Indeed, the finding from the present study that the methylation ratios of CpG3 and CpG22:23:24 were positively associated with olfactory score provide a pointer for further investigation to test this hypothesis. Similarly, our finding that hypermethylation at CpG3 and CpG22:23:24 was highly correlated with unilateral nasal resistance rather than total nasal resistance raises the possibility that TSLP may also be involved in the aetiology of nasal obstruction, which is one of the most common symptoms in CRS patients. Currently, nasal airflow resistance is frequently used for the objective evaluation of rhinostenosis [34]. The results in the present study provided evidence that hypermethylation at CpG3 and CpG22:23:24 might have effects on clinical phenotypes, including hyposmia and nasal obstruction, in the pathophysiology of CRS.

However, the findings of this study are slightly limited, particularly in view of differences in some baseline demographic characteristics of subjects in the CRSwNP group compared to the CRSsNP and control groups. Thus, given that the baseline demographic for age was statistically significantly higher in patients with CRSwNP than in control and CRSsNP patients; and multivariate analysis also demonstrated that, apart from the disease status, only age significantly influenced the methylation ratio of specifically CpG3 site within the TSLP promoter, it is possible that the observed effects on methylation within the TSLP promoter may at least partly be agerelated. Indeed, it is likely that the significant difference noted for the baseline demographic of higher olfactory scores in patients with CRSwNP than in control and 
CRSsNP patients, was also age-related, because olfactory function declines with age. Furthermore, this study is also somewhat limited by the relatively small sample size, and therefore these findings need to be validated in larger age-matched cohorts. Moreover, the levels of TSLP protein and receptor in nasal mucosa or nasal secretions were not measured, as increased levels of these proteins in CRSwNP patients would have provided some support for the present findings for the relationship between methylation status of TSLP and CRSwNP.

\section{Conclusion}

In conclusion, this preliminary study provides new insight into the role of TSLP in the development of CRS and shows that increased DNA methylation at the TSLP locus is likely to be associated with CRSwNP pathogenesis; however these findings need to be confirmed in multicentre studies with larger age-matched cohorts. Further, although bioinformatics databases predict that CpG3 and CpG22:23:24 units can disrupt binding of several transcriptional factors, experimental evidence of the functional effects of methylation in these sites is still lacking. Nevertheless, this approach may be useful for further investigation of the aetiology of CRS to fill the gap of genetic and non-epigenetic mechanisms.

\section{Supplementary information}

Supplementary information accompanies this paper at https://doi. org/10.1186/s13223-019-0389-3.

Additional file 1: Table S1. Positions and sequences of primers used for the EpiTYPER assay to analyze DNA methylation of TSLP locus. Figure S1. Schematic representation of the human TSLP locus on UCSC Genome Browser (hg19).

\section{Abbreviations}

AR: allergic rhinitis; AD: atopic dermatitis; CRS: chronic rhinosinusitis; CRSwNP: CRS with nasal polyps; CRSsNP: CRS without nasal polyps; DCs: dendritic cells; FDR: false discovery rate; HNECs: human nasal epithelial cells; IT: inferior turbinate; SNPs: single nucleotide polymorphisms; TSLP: thymic stromal lymphopoietin.

\section{Acknowledgements}

Not applicable.

\section{Authors' contributions}

$L, Z Y$ and $Z L$ designed the experiments, performed the data analyses and drafted the manuscript. $L J, J$ J and GY contributed to experiments management and data collection. All authors read and approved the final manuscript.

\section{Funding}

This work was supported by grants from the national key R\&D program of China (2016YFC20160905200), the program for the Changjiang scholars and innovative research team (IRT13082), the National Natural Science Foundation of China (81420108009, 81630023, 81970849 and 81570895), Beijing Natural Science Foundation (7182034 and 7172053), Beijing municipal administration of hospitals' mission plan (SML20150203), and Beijing advanced innovation centre for food nutrition and human health (Beijing Technology and Business
University, 20181045), Beijing Health Bureau Program for High Level Talents (2014 -3-018) and Cross-disciplinary Collaborative Program of Beijing Nova program (xxjc201712).

\section{Availability of data and materials}

We would like to provide the raw data to support the information presented in this publication.

\section{Ethics approval and consent to participate}

The study was approved by the Ethics Committee of Beijing Tongren Hospital and all subjects provided written informed consent prior to recruitment.

\section{Consent for publication}

Not applicable.

\section{Competing interests}

The authors declare that they have no competing interests.

\section{Author details}

${ }^{1}$ Department of Otolaryngology Head and Neck Surgery, Beijing TongRen Hospital, Capital Medical University, Beijing 100730, China. ${ }^{2}$ Beijing Key Laboratory of Nasal Diseases, Beijing Institute of Otolaryngology, No. 17 HouGouHuTong, DongCheng District, Beijing 100005, China. ${ }^{3}$ Department of Allergy, Beijing TongRen Hospital, Capital Medical University, Beijing 100176, China.

Received: 10 June 2019 Accepted: 12 November 2019 Published online: 21 November 2019

\section{References}

1. Hamilos DL. Chronic rhinosinusitis: epidemiology and medical management. J Allergy Clin Immunol. 2011;128(4):693-707 (quiz 708-699).

2. Jarvis D, Newson R, Lotvall J, Hastan D, Tomassen P, Keil T, et al. Asthma in adults and its association with chronic rhinosinusitis: the GA2LEN survey in Europe. Allergy. 2012;67(1):91-8.

3. Shi JB, Fu QL, Zhang H, Cheng L, Wang YJ, Zhu DD, et al. Epidemiology of chronic rhinosinusitis: results from a cross-sectional survey in seven Chinese cities. Allergy. 2015;70(5):533-9.

4. Yang IV, Lozupone CA, Schwartz DA. The environment, epigenome, and asthma. J Allergy Clin Immunol. 2017;140(1):14-23.

5. $\mathrm{Ng} \mathrm{HH}$, Bird A. DNA methylation and chromatin modification. Curr Opin Genet Dev. 1999;9(2):158-63.

6. Bert SA, Robinson MD, Strbenac D, Statham AL, Song JZ, Hulf T, et al. Regional activation of the cancer genome by long-range epigenetic remodeling. Cancer Cell. 2013;23(1):9-22.

7. Lee DD, Leao R, Komosa M, Gallo M, Zhang CH, Lipman T, et al. DNA hypermethylation within TERT promoter upregulates TERT expression in cancer. J Clin Invest. 2019;129(1):223-9.

8. Martino D, Kesper DA, Amarasekera M, Harb H, Renz H, Prescott S. Epigenetics in immune development and in allergic and autoimmune diseases. J Reprod Immunol. 2014;104-105:43-8.

9. Soumelis V, Reche PA, Kanzler H, Yuan W, Edward G, Homey B, et al. Human epithelial cells trigger dendritic cell mediated allergic inflammation by producing TSLP. Nat Immunol. 2002;3(7):673-80.

10. Nagarkar DR, Poposki JA, Tan BK, Comeau MR, Peters AT, Hulse KE, et al. Thymic stromal lymphopoietin activity is increased in nasal polyps of patients with chronic rhinosinusitis. J Allergy Clin Immunol. 2013;132(3):593-600.

11. Kimura S, Pawankar R, Mori S, Nonaka M, Masuno S, Yagi T, et al. Increased expression and role of thymic stromal lymphopoietin in nasal polyposis. Allergy Asthma Immunol Res. 2011;3(3):186-93.

12. Liao B, Cao PP, Zeng M, Zhen Z, Wang H, Zhang YN, et al. Interaction of thymic stromal lymphopoietin, IL-33, and their receptors in epithelial cells in eosinophilic chronic rhinosinusitis with nasal polyps. Allergy. 2015;70(9):1169-80

13. Hunninghake GM, Soto-Quiros ME, Avila L, Kim HP, Lasky-Su J, Rafaels N, et al. TSLP polymorphisms are associated with asthma in a sex-specific fashion. Allergy. 2010;65(12):1566-75. 
14. Margolis DJ, Kim B, Apter AJ, Gupta J, Hoffstad O, Papadopoulos M, et al. Thymic stromal lymphopoietin variation, filaggrin loss of function, and the persistence of atopic dermatitis. JAMA Dermatol. 2014;150(3):254-9.

15. Bunyavanich S, Melen E, Wilk JB, Granada M, Soto-Quiros ME, Avila L, et al. Thymic stromal lymphopoietin (TSLP) is associated with allergic rhinitis in children with asthma. Clin Mol Allergy. 2011;9:1.

16. Zhang $Y$, Wang $X$, Zhang W, Han D, Zhang L, Bachert C. Polymorphisms in thymic stromal lymphopoietin gene demonstrate a gender and nasal polyposis-dependent association with chronic rhinosinusitis. Hum Immunol. 2013;74(2):241-8.

17. Fokkens WJ, Lund VJ, Mullol J, Bachert C, Alobid I, Baroody F, et al. European position paper on rhinosinusitis and nasal polyps 2012. Rhinology. 2012;23:3.

18. Ren L, Zhang L, Duan S, Zhang W, Zhang Y. Nasal airflow resistance measured by rhinomanometry in a healthy population of China. Int Forum Allergy Rhinol. 2018;8(11):1308-14.

19. Jiao J, Duan S, Meng N, Li Y, Fan E, Zhang L. Role of IFN-gamma, IL-13, and IL-17 on mucociliary differentiation of nasal epithelial cells in chronic rhinosinusitis with nasal polyps. Clin Exp Allergy. 2016;46(3):449-60.

20. Ziegler SF. Thymic stromal lymphopoietin and allergic disease. J Allergy Clin Immunol. 2012;130(4):845-52.

21. Watanabe N, Wang YH, Lee HK, Ito T, Wang YH, Cao W, et al. Hassall's corpuscles instruct dendritic cells to induce $\mathrm{CD}^{+} \mathrm{CD} 25^{+}$regulatory $\mathrm{T}$ cells in human thymus. Nature. 2005;436(7054):1181-5.

22. Hanabuchi S, Ito T, Park WR, Watanabe N, Shaw JL, Roman E, et al. Thymic stromal lymphopoietin-activated plasmacytoid dendritic cells induce the generation of $\mathrm{FOXP3} 3^{+}$regulatory $\mathrm{T}$ cells in human thymus. J Immunol. 2010;184(6):2999-3007

23. Watanabe N, Hanabuchi S, Soumelis V, Yuan W, Ho S, de Waal Malefyt $R$, et al. Human thymic stromal lymphopoietin promotes dendritic cell-mediated CD4 ${ }^{+}$T cell homeostatic expansion. Nat Immunol. 2004;5(4):426-34.

24. Ito T, Wang YH, Duramad O, Hori T, Delespesse GJ, Watanabe N, et al. TSLP-activated dendritic cells induce an inflammatory T helper type 2 cell response through OX40 ligand. J Exp Med. 2005;202(9):1213-23.

25. Fornasa G, Tsilingiri K, Caprioli F, Botti F, Mapelli M, Meller S, et al. Dichotomy of short and long thymic stromal lymphopoietin isoforms in inflammatory disorders of the bowel and skin. J Allergy Clin Immunol. 2015;136(2):413-22.

26. Bjerkan L, Schreurs O, Engen SA, Jahnsen FL, Baekkevold ES, Blix IJ, et al. The short form of TSLP is constitutively translated in human keratinocytes and has characteristics of an antimicrobial peptide. Mucosal Immunol. 2015;8(1):49-56.

27. Luo Y, Zhou B, Zhao M, Tang J, Lu Q. Promoter demethylation contributes to TSLP overexpression in skin lesions of patients with atopic dermatitis. Clin Exp Dermatol. 2014;39(1):48-53.

28. Dong H, Hu Y, Liu L, Zou M, Huang C, Luo L, et al. Distinct roles of short and long thymic stromal lymphopoietin isoforms in house dust mite-induced asthmatic airway epithelial barrier disruption. Sci Rep. 2016:6:39559.

29. Lan F, Zhang N, Holtappels G, De Ruyck N, Krysko O, Van Crombruggen $\mathrm{K}$, et al. Staphylococcus aureus induces a mucosal type 2 immune response via epithelial cell-derived cytokines. Am J Respir Crit Care Med. 2018;198(4):452-63.

30. Pozharskaya T, Lane AP. Interferon gamma causes olfactory dysfunction without concomitant neuroepithelial damage. Int Forum Allergy Rhinol. 2013;3(11):861-5.

31. Turner JH, May L, Reed RR, Lane AP. Reversible loss of neuronal marker protein expression in a transgenic mouse model for sinusitis-associated olfactory dysfunction. Am J Rhinol Allergy. 2010;24(3):192-6.

32. Turner JH, Liang KL, May L, Lane AP. Tumor necrosis factor alpha inhibits olfactory regeneration in a transgenic model of chronic rhinosinusitisassociated olfactory loss. Am J Rhinol Allergy. 2010;24(5):336-40.

33. Wilson SR, The L, Batia LM, Beattie K, Katibah GE, McClain SP, et al. The epithelial cell-derived atopic dermatitis cytokine TSLP activates neurons to induce itch. Cell. 2013;155(2):285-95.

34. Eccles R. Nasal airflow in health and disease. Acta Otolaryngol. 2000;120(5):580-95.

\section{Publisher's Note}

Springer Nature remains neutral with regard to jurisdictional claims in published maps and institutional affiliations.
Ready to submit your research? Choose BMC and benefit from:

- fast, convenient online submission

- thorough peer review by experienced researchers in your field

- rapid publication on acceptance

- support for research data, including large and complex data types

- gold Open Access which fosters wider collaboration and increased citations

- maximum visibility for your research: over $100 \mathrm{M}$ website views per year

At BMC, research is always in progress.

Learn more biomedcentral.com/submissions 